\title{
Quantifying 3D micro-surface changes on experimental stones used to break bones and their implications to the analysis of Early Stone Age pounding tools
}

Alfonso Benito-Calvo ${ }^{1}$, Adrián Arroyo ${ }^{2}$, Laura Sánchez- Romero ${ }^{1}$, Michael Pante ${ }^{3}$, Ignacio de la Torre Tै $^{2 *}$

\footnotetext{
${ }^{1}$ Laboratory of Digital Mapping and 3D Analysis, CENIEH, Paseo Sierra de Atapuerca, 3, 09002 Burgos, Spain

${ }^{2}$ Institute of Archaeology, University College London. 31-34 Gordon Square, WC1H OPY. London. UK

${ }^{3}$ Department of Anthropology, Colorado State University, Fort Collins, Colorado, 80523, USA

*Corresponding author: i.torre@ucl.ac.uk (I. de la Torre)
}

\begin{abstract}
We present a new method to assess use-wear formation processes of pounding tools used to break bones based on a combination of conventional microscopy, optical 3D surface measurements obtained with a confocal microscope and GIS analysis. The method involves 3D alignment and 3D surface change inspection techniques along with a surface morphometric characterization and 2D spatial pattern analysis, to measure the spatial distribution of significant changes in surface topography of pounding tools. Our results show that microscopic changes can be detected in the surfaces of hammers and anvils after bone breakage activities are performed. Use-wear on the active elements (hammers made on basalt and quartzite) occurred over a larger area than was observed on the passive element (quartzite anvil), but the latter often exhibited deeper modifications. Tool surfaces generally developed smoother topography with increased use, but grain microfracture also appeared with greater frequency over time. This methodology offers highly accurate and statistically robust analyses of microscopic use-wear traces that can be applied to the analysis of archaeological pounding tools.
\end{abstract}




\section{Keywords}

Experimental archaeology, use-wear, 3D surface analysis, pounding tools, GIS.

\section{Introduction}

Semenov's (1964) groundbreaking work to assess the functionality of prehistoric stones tools through microscopic analysis prompted decades of research on the study and quantification of use-wear. Originally, use-wear analyses were based on direct observations and description of polish, linear traces or microfractures with binocular, metallographic or scanning electron microscopes (SEM) (Semenov, 1964; Keller, 1966; Frison, 1968, Tringham et al., 1974; Keeley, 1980; Anderson-Gerfaud, 1981; Odell, 1981; Mansur-Franchomme, 1983; Vaughan, 1985; Plisson, 1985; Knutsson, 1988; Hurcombe, 1992; Vergès and Ollé, 2011; Ollé and Vergès, 2014, and references therein).

However, difficulties in quantifying use-wear traces with optical technologies were recognized early on, and researchers sought alternative means to measure these microscopic traces (Keeley, 1980, Grace et al., 1985, Beyries et al., 1988). As a result, multiple techniques were developed to reliably quantify use-wear traces, with most relying on observation of differences in the intensity of reflected light related to changes in the texture of the rock surfaces (Barceló et al., 2001; Toselli et al., 2002; Gonzalez-Urquijo and Ibáñez-Estévez, 2003), and others using the RIMAPS (rotated image with maximum average power spectrum) method (Álvarez et al., 2012) and focus variation microscopy (Macdonald, 2014).

Researchers have also applied advanced technologies such as atomic force and confocal microscopes to measure surface roughness through fractal analysis (Kimball et al., 1998; Anderson et al., 1998; Stemp and Stemp, 2001; 2003; Stemp et al., 2013; 2015; 2016; Stevens et al., 2010; Evans and Donahue, 2008), a technique that is sensitive to small changes in surface topography and can used to quantify use-wear traces on archaeological tools 
(Ibáñez et al., 2016). Most recently, GIS techniques have been employed to analyze 3-D data generated with these technologies (de la Torre et al., 2013; Caruana et al., 2014; BenitoCalvo et al., 2015).

In this paper, we integrate standard low magnification methods based on binocular microscopy with confocal optical microscopy to provide high-resolution 3D measurements and quantitative observations aimed at characterizing use-wear resulting from hammerstoneon-anvil breakage of bones. Previous studies have compared two topographic surfaces surveyed before and after the studied process (Abellán et al., 2010; Schürch et al., 2011; Lague et al., 2013)-, but have not been applied to measure the progression of changes to the surface topography of stone tools. Analytical techniques employed here include alignment, surface change inspection, and morphometric characterization of use-wear, followed by a 2D spatial pattern analysis to characterize the spatial distribution of changes. Our aim is to provide accurate and replicable results that are statistically robust at both meso- and microscales.

The approach presented here is designed to afford interpretations of use-wear on Early Stone Age pounding tools. It builds on previous methods aimed at connecting use-wear traces on pounding tools from Olduvai Gorge to those produced by pounding activities in controlled experiments (Mora and de la Torre, 2005; de la Torre et al., 2013; Sánchez Yustos et al., 2015; Arroyo and de la Torre, 2016). The high-resolution data collected here through optical confocal microscopy, allows refinement of the quantification of use-wear traces at a microscopic scale, which was not possible with the lower resolution methods of 3D data collection previously employed.

\section{Materials and methods}

\subsection{Experimental settings}


Previous experimental programs have stressed the value of sequential experiments and continuous observation to get a detailed record of use-wear formation processes beginning with an unmodified rock surface (Yamada, 1993; Ollé and Vergès, 2014). We have applied this concept to study use-wear on pounding tools that were used to break limb bones with the goal of extracting marrow.

Our experiments included three pounding tools: one tabular quartzite anvil (ID: O98 measuring 182 × 134 × $74 \mathrm{~mm}$ and $2369.3 \mathrm{~g}$ ) and two hammerstones: a quartzite cobble (ID: O86 measuring $72 \times 67 \times 69 \mathrm{~mm}$ and $511.8 \mathrm{~g}$ ) and a basalt cobble (ID: O83 measuring 129 x 103 x $70 \mathrm{~mm}$ and $1045.8 \mathrm{~g}$ ). Quartzite was collected from Naibor Soit, the main hominin raw material source at Olduvai Gorge (Tanzania) during the Lower Pleistocene, while the basalt cobble was recovered from the modern riverbed at Olduvai.

Prior to the experiments, specific areas of the tools were selected to be used as control points $(\mathrm{CP})$, where repeated observations were made to document the progression of usewear. The state of the unused surfaces for each $\mathrm{CP}$ were documented microscopically at various magnifications $(20 \times, 30 \times, 45 \times)$ (Figure 1C). A microscopic inspection of the areas selected on each tool prior to their use, did not reveal any traces that would be identified as use-wear. Specifically, the quartzite anvil did not have fresh fractures or polish in its CP, while both hammerstones lacked fluvial cortex with uniform grain distributions and a low degree of surface polish.

The majority of microscopic analyses were conducted using a GX stereo-microscope, (magnification range of $0.75-4.5 \times$ ) with a $10 \times$ ocular lens, and a Nikon D90 camera attached for recording images. However, after the final stage of the experiments, we used a Leica S8APO microscope (magnification range of $1 \times-8 \times$ ), equipped with a $10 \times$ ocular lens, an EC3 3.1-megapixel digital camera and fiber optic illumination, for its superior magnification. The 3D microscopic details of the CPs were captured with a NanoFocus $\mu$ Surf Mobile Plus 
Micro-3D scanner equipped with an Olympus $160010 \times$ objective $(10 \times / 0.30)$. The scanner employs a LED light source, a rotating multi-pinhole disc, an objective lens and CCD camera to collect surface data (Konschak, 2012). CP's were scanned individually at a working distance of $11.00 \mathrm{~mm}$, a pitch of $0.937 \mu \mathrm{m}$ and at the medium speed setting ( 3 out of 5).

The quartzite anvil had four CPs recorded with the microscopic and three CPs that were 3D scanned. The quartzite hammerstone had two CPs that were recorded with the optical microscopes and two with the micro-3D scanner, while the basalt hammerstone had three CPs recorded with the microscopes and two with the 3D scanner.

A total of 20 defleshed cow limb bones ( 3 tibiae and 17 femora) were processed in the experiments, which were carried out over multiple sessions so that data could be collected and the progression of use-wear assessed. The number of bones that were broken in each session varied, but was never more than four (Table S1). Hammerstone-on-anvil breakage of bones followed methods prescribed by Blumenschine (1988), with the goal of splitting bones longitudinally along their axes to facilitate the extraction of marrow. Bones were placed directly over the CP's on the anvil, which were located along one edge. They were struck on either the medial or lateral surfaces at three general locations along the diaphysis (proximal shaft, mid shaft and distal shaft) (Figure 1). Bones were moved so that the anvil CP was directly opposite the point of hammerstone impact, while hammerstones were held so that the CPs were directly impacting the bone surface. Each experimental session was recorded on video for further analysis.

\section{Insert Figure 1}

After each experiment, pounding tools were washed to eliminate fat residues. They were rinsed with water, left to soak overnight in a neutral soup solution, subsequently cleaned 
in an ultrasonic bath for 10 minutes, and rinsed again with water. This process was repeated until all macroscopic residues were removed. All CPs were photographed and scanned after each session with the techniques described above.

\subsection{D analytical techniques}

Microscopic changes on the pounding tools were assessed with multiple variables including distance changes, slope, plan and profile curvature, and two different roughness indices: TRI (Terrain Ruggedness Index) and VRM (Vector Ruggedness Measure). These indices have been previously tested and positively applied at a macroscopic scale in the analysis of pounding tools used by wild chimpanzees (Benito-Calvo et al., 2015). The data was processed using the open access software CloudCompare (Girardeau-Montaut, 2015), and SAGA GIS (Conrad et al., 2015).

\subsubsection{D alignment}

In order to guarantee a robust and fine morphometric surface comparison, the successive 3D point clouds measured in each $\mathrm{CP}$ were georeferenced in a common spatial coordinate system. Georeferencing can be done at macro- or meso-scales using preestablished reference points (e.g. ground control points or targets). To define accurate reference points at microscales when surface topography is under modification can be a fruitless task. However, 3D alignment techniques can alleviate this problem by matching multiple 3D entities and minimizing the differences between the 3D point clouds.

We used the Iterative Closest Point (ICP) technique (Zhang, 1994; Girardeau-Montaut et al., 2015) for registering the micro point clouds in a common coordinate system for each CP. Through this technique, a reference point cloud is kept fixed, while a second point cloud is transformed (usually by a combination of translation and rotation) to best match the reference point cloud (Girardeau-Montaut et al., 2015). The ICP algorithm iteratively recalculates the transformation needed to minimize the error from the transformed to the 
reference point cloud. 3D point clouds were measured before and after the percussion activity for each control point (CP), and then they were registered in a common coordinate system. The latter was applied using a random sample always greater than 100,000 comparison points (Table S2).

During registration, the original scale of each 3D point cloud was preserved. Resulting registration errors were defined by RMS between 3 and 4 microns (Table S2). These registration errors were considered as acceptable, as they are close to the XY spatial resolution of the point clouds (3.125 microns). 3D alignment techniques require substantial overlap between CPs scans in order to achieve a representative analysis area and the best 3D alignment. This was only possible for two CPs (O83/CP1 ST3, O98/CP2 stages ST3 and ST6). The required common geometries of CPs could not be overlapped for O86.

\subsubsection{Significant distance changes}

Once the point clouds were registered, we assessed changes in surface topography that occurred after each session of percussion activity by comparing the point clouds measured before and after the pounding session. This comparison provides a distance, which represents the perpendicular separation between the two topographic surfaces for each point, and can be transformed into volume units considering the resolution or XY spacing of the 3D confocal models. Topographic surfaces can be expressed in several ways, from simply XYZ data to more elaborate formats. The simplest formats are composed of discontinuous data, such as 3D points forming point clouds, or contours. More complex formats such as Digital Elevation Models (DEM) or vectorial meshes composed by triangulation, produce continuous topographic data. Surface comparisons are usually based on DEM of difference (DoD) (Abellán et al., 2010; Schürch et al., 2011), cloud-to-mesh or cloud-to-model comparisons (C2M) (Monserrat and Crosetto, 2008; Olsen et al., 2009), and direct cloud-to-cloud comparison (C2C) (Girardeau-Montaut et al., 2015). Techniques based on model generation 
(DoD or $\mathrm{C} 2 \mathrm{M}$ ) come with drawbacks inherent to the surface meshing or the DEM generation (i.e. time consuming, resolution, surface roughness, noise effects, etc.). The $\mathrm{C} 2 \mathrm{C}$ technique provides the simplest and fastest direct 3D comparison, although it is also sensitive to surface roughness, outliers and point spacing; and does not include signed data or confidence intervals in the calculation (Girardeau-Montaut et al., 2015).

The algorithm used here to detect topographical changes in the micro-surfaces of CPs after the percussion activity is the M3C2, developed by Lague et al. (2013). In comparison with other methods, the $\mathrm{M} 3 \mathrm{C} 2$ algorithm has been demonstrated to be more accurate (Lague et al., 2013), and has as an easier workflow due to the absence of surface meshing or Digital Elevation Model (DEM) generation. This algorithm provides signed and robust separation distances, obtained directly from the comparison of two 3D point clouds. M3C2 works in two steps defined by 1) the surface normal estimation in 3D at a scale consistent with the local surface roughness, and 2) the measurement of the mean surface change along the normal direction with explicit calculation of a local confidence interval. The latter attempts to capture the effects of surface roughness, position uncertainty and registration error, in order to detect statistically significant changes at a 95\% confidence interval. Parameters used for the M3C2 calculation are detailed in Table S3.

\subsubsection{Spatial pattern of significant changes}

Areas that exhibited statistically significant changes can be processed as categorical maps, which can be used to investigate the spatial pattern of changes. De la Torre et al. (2013) provided a set of variables to assess spatial distributions, which are mainly based on the calculation of the area, perimeter, size and shape of the areas showing changes. The distribution of features was estimated with the standard deviational ellipse of the distribution (de la Torre et al., 2013), which measures the directional trend, shape and relative position of the features considered. 


\subsubsection{Morphometric changes}

Point cloud comparisons allow assessment of surface changes through distance calculation, but other morphometric variables can also be used to describe surface topographic changes and recognize use-wear features (Benito-Calvo et al., 2015). In order to analyze other morphometric changes across the surface, point clouds were gridded into DEMs, from which we calculate slope, curvature and roughness variables in a Geographical Information System (Conrad et al., 2015). Gridding was performed using the spatial resolution of data captured with confocal microscopy $(3.125 \mu \mathrm{m})$.

\section{Results}

\subsection{Macroscopic and microscopic analysis}

Percussive artefacts showed very little evidence of modification at the macroscopic scale with a total absence of fractures or other modification to their general form. Only the quartzite hammerstone (O86) developed percussive wear traces that could be recognized macroscopically according to criteria defined by de la Torre et al. (2013), therefore we will focus on the microscopic approach.

The microscopic analysis of the pounding tools after each experimental session showed that the formation of use-wear was slow. For example, despite being used in all experimental sessions and receiving a total of 520 indirect strikes, the anvil (O98) exhibited only minimal observable modification (Figure 2) that included an isolated small shallow, a superficial area of crushed crystal located near CP-C (Figure 2-B7) and a possible area of abrasion (sensu Keeley, 1980; Sussman, 1988) near CP-D. There were not any missed blows, and therefore, there was no direct contact between the active and the passive element, which has been reported to be a main cause of use-wear formation (de la Torre et al., 2013). The low degree of modification to the passive anvil is consistent with previous observations of 
bone breaking experiments (de la Torre et al., 2013; Sánchez Yustos et al., 2015) and is likely the result of the majority of the force transmitted by the hammerstone getting absorbed by the bone. Use-wear formed on the anvil may be more related to an abrasive process caused by the movement of the bone across the surface during the activity. Damage resulting from compressive forces was limited to weaker areas located on the edges of the anvil, where microscopic fracturing developed at the point of contact with the bones.

\section{Insert Figure 2}

The active elements showed more obvious modification after use, with the quartzite hammerstone exhibiting greater damage than the basalt. The quartzite hammerstone (O86) was used to process 10 bones and delivered 276 strikes. Percussive wear traces on O86 started to develop after the first set of 3 bones (ST1), beginning with superficial crystal crushing (Figure 3-A2 and 3-B2). This damage became more intense as the experiments progressed (Figure 3A-3 and 4; 3B-3 and 4), producing detachment of small crystal fragments and the appearance of scattered pits (Figure 3A-5). After the final session, usewear traces were similar to battering produced on hammerstones through the production of stone flakes from a core. The lava hammerstone (O83) was used to process 10 bones, delivering a total of 244 strikes. This active element showed more superficial modifications than the O86, with a complete lack of deep batter marks. Damage was limited to scattered impact areas and small micro-fractures on the edges of some of the natural vesicles, but no large areas of grain crushing or other conspicuous marks were apparent under the low magnification used here (Figure 4). The wear traces identified on the active elements are similar to what is called 'mechanical wear' in tribology (sensu Kato, 2002), a process which is dominated by the fracturing of crystals and grains. The elasticity of bone likely slowed and 
minimized the development of use-wear on the more impact resistant stone.

Insert Figure 3

Insert Figure 4

\subsection{Micro-3D surface modifications}

\subsubsection{Morphometric analysis before the 3D alignment}

The low degree of surface modification to the pounding tools after the experiments is also evident when looking at the data obtained with the micro-3D scanner. The use of this technology allows a more detailed and quantifiable description of the damage. For example, the mean curvature of the surface increased at both CPs in tool O86, especially at the last stage (ST3) of the experiments (Figure S1). This supports the microscopic analysis, which showed the use-wear damage increased over time. Additionally, one of the CPs on the anvil (CP2), showed an increase to the curvature of the surface that was observable with the micro3D scanner, but not with the stereo microscopes.

A morphometric analysis of every $\mathrm{CP}$ was initially conducted using the raw data (i.e. before the $3 \mathrm{D}$ alignment). In this case, we measured changes to the mean values of the TRI and VRM roughness indices, both of which showed significant changes (Figure S2). These results indicate a fracturing/crushing and then levelling wear formation pattern for the hammerstones, especially O86, but not the anvil.

For O86, in the first stage of use (ST1), there is a decrease in surface roughness (VRM and TRI indices). In ST2 roughness increases, before decreasing again in ST3. This pattern has also been observed through microscopic analysis of pounding tools. In the initial stage, surfaces are levelled with high points getting removed, thus roughness decreases; in the 
second stage, the crystal structure becomes weaker and is ultimately crushed resulting in micro-fracturing and an increase in roughness; in the third stage, micro-fractured areas become levelled and roughness decreases again. A similar pattern was observed for hammerstone $\mathrm{O} 83$, but the process began with an increase in roughness, probably because the natural surfaces of lavas tend to be more regular or smoother than the quartzite.

The anvil (O98) did not show this pattern of alternating increases and decreases to roughness in either the micro-3D or microscopic analyses. However, the TRI and VRM indices generally decrease towards the end of the experiments (Figure S2), possibly due to micro levelling of the surface making the surface smoother, a process that could not be observed with a low magnification approach.

\subsubsection{Morphometric analysis of 3D aligned data}

Morphometric analysis of georeferenced data detected in control points for O83 and O98 are summarized in Table 1 . These changes are characterized by a general decrease in the mean elevation. Apart from this logical trend in surface modification resulting from use, we have also found other less obvious patterns. An increase in the mean slope and the mean roughness values were detected between the unused state and stage 3 for CP1 of O83 and CP2 of O98. This pattern was reversed between stages 3 and 6 for CP2 of O98. The surface curvature, profile and plan curvatures show different trends. While profile curvature increases in all the stages of the percussion activity, plan curvature falls from the unmodified stage to stage 3 in both pieces (O83 and O98), and rises from stage 3 to stage 6 in piece O98. These results were mirrored in a third $\mathrm{CP}$ for piece $\mathrm{O} 98$ (98 CP3), showing the same general morphometric trends observed in CP2 of O98.

\section{Insert Table 1}




\subsubsection{Significant distance changes}

Changes in the CPs of $\mathrm{O} 83$ and $\mathrm{O} 98$ were assessed by measuring the separation distance between the registered 3D point clouds using the M3C2 parameters defined in Table 3 (Lague et al., 2013). This detects areas where the surface has been altered.

Changes detected in $83 \mathrm{CP} 1$ are shown in Figure 5A and B, and demonstrate that this $\mathrm{CP}$ has changed significantly over $9.7 \%$ of the measured surface (Table 2A). $99.1 \%$ of the distance values decreased (Table 2B), indicating removal of the surface through use of the hammerstone. Decreases in distance values range from -7 to $-42 \mu \mathrm{m}$, with a peak at $-8 \mu \mathrm{m}$ (Figure 5C). In total, the volumetric loss of the measured area is $-1,709,704 \mu \mathrm{m}^{3} .0 .9 \%$ of the total significant changes are positive (Table 2B), suggesting areas where the surface topography has somehow been built up (Figure 5A and C). The amount of buildup ranges from $6.4 \mu \mathrm{m}$ to a maximum of $11 \mu \mathrm{m}$, with a mean of $7 \mu \mathrm{m}$ (Figure $5 \mathrm{C}$ ) and a volume increase of $9284 \mu \mathrm{m} 3$.

\section{Insert Figure 5}

\section{Insert Table 2}

There are two phases considered for 98 CP2, with UNM-ST3 and ST3-ST6 representing the third and the last (sixth) stage of use (Figure 5D to G). Only $6.1 \%$ of the measured area has significant surface changes for UNM-ST3. $61.5 \%$ of the change is a decrease in distance values (Table 2A), which ranges from $-7.9 \mu \mathrm{m}$ to $-79.1 \mu \mathrm{m}$ (Figure $5 \mathrm{D}$ to $\mathrm{G})$. Changes in this range show a multimodal pattern defined by a main mode located at -10 $\mu \mathrm{m}(22.63 \%$ of the negative change $)$ and minor peaks at $-21 \mu \mathrm{m}(2.48 \%)$ and $-30 \mu \mathrm{m}(1.26 \%)$ (Figure 5C). The total negative volumetric change was measured at $-1,227,660 \mu \mathrm{m} 3$. 
Increases in the distance values were also observed (Figure 5D to $\mathrm{G}$ ), representing a greater proportion of the significantly modified surface relative to $83 \mathrm{CP} 1$. Specifically, positive distance values correspond to the $34.1 \%$ of the significant changes (Table 2B), representing a volumetric increase of $662,732 \mu \mathrm{m} 3$. This increment shows a distribution dominated by a mean peak at $9.7 \mu \mathrm{m}$ (Figure $5 \mathrm{C}$ ).

ST3-ST6 also shows a low percentage (6.1\%) of the surface is significantly changed (Table 2A, Figure 5E and G). However, decreases in distance values are more common at $89.3 \%$, with volumetric changes of $-2,527,253 \mu \mathrm{m} 3$ (Figure 5C). Negative distance values fall between $-7 \mu \mathrm{m}$ and $-117 \mu \mathrm{m}$. The distribution of negative distance values entails a main mode at $9 \mu \mathrm{m}$, representing $14.4 \%$ of the negative change (Figure $5 \mathrm{C}$ ), and a longer tail with minor peaks at $-25 \mu \mathrm{m}(2.92 \%)$ and $44 \mu \mathrm{m}(1.6 \%)$. In this control point, significant increases in distance values were also observed (10.7\%) and range between 7 and $80 \mu \mathrm{m}$, showing a distribution dominated by a peak at $8.4 \mu \mathrm{m}$ (Figure 5C). The volume of positive significant changes to distance values is $143,443 \mu \mathrm{m}^{3}$.

\subsubsection{Spatial pattern of significant changes}

Table 6 summarizes the results of the changes to controls points O83-CP1/ST3, O98CP2/ST3 and ST6. These quantitative results support the qualitative observations made with the stereo microscope. The hammerstone (O83) showed the greatest percentage of significantly modified surface $(\mathrm{PA}=9.65 \%)$, the biggest individual modified area $(\mathrm{LUW}=1.56 \%)$ and modifications that tend to be more irregular in shape (Elongation=1.24). 3D models of the anvil have revealed the highest densities of impact marks $(D=0.00016$ $\mathrm{n} / \mu \mathrm{m}^{2}$ ) despite an absence of microscopic use-wear traces when observed with a stereo microscope. The spatial distribution of significant changes showed similar patterns, defined by deviational ellipses located in a centered position and with similar elongation characteristics in a vertical or subvertical position (Table $\mathrm{S} 4$, Figure $5 \mathrm{H}$ ). 


\section{Discussion}

\subsection{The identification of surface modification using 3D models}

3D alignment techniques are effective at registering correlative point clouds in a common reference system, which is a critical step to reliably assess changes in surface morphology. This process is applicable to large sets of comparison points $(>100,000)$, which provide accurate and robust results (Table S2). Despite this success, the analyses were not without problems. While the ICP algorithm was effective at establishing a common georeferenced system of point clouds for pieces $\mathrm{O} 83$ and O98, a point cloud for piece O86 could not be registered. We did not find common geometries to apply the ICP algorithm in the control point of this piece likely because the percussive activity completely wore down the surface around the CPs. This indicates a softer lithology and/or a more intensive use for piece O86, and also suggests 3D alignment techniques may not be applicable when surface changes are extreme. However, this limitation could be minimized by creating larger control points that include more unmodified surface in the initial 3D data capture.

The statistically significant changes measured ranged between 7 and $117 \mu \mathrm{m}$ (Figures 5), values that are much higher than the registration errors indicating the reliability of measured variables even at micro-scales. These measures allow for the quantification of surface changes in ways that are not possible using data collected with a stereo microscope. For example, we can compare the volume of change in surface topography noting that the change was much greater in the hammerstone O83 than the anvil O98. This indicates than the modified surface area is greater in the hammer than in the anvil (Figure S3). We can also determine that the maximum change in depth is greater in the anvil at $-111 \mu \mathrm{m}$ than the hammerstone at $-42 \mu \mathrm{m}$.

We observed both decreases and increases in distance values, which quantify changes in surface morphology. Most of the changes we observed were decreases in the measured 
distance values resulting from impacts of the hammerstone on the bone or the bone on the anvil. However, we also detected statistically significant increases to distance values, which were likely the result of a buildup of tissue that was transferred from bones to stones during breakage. This was more significant in the hammerstone than in the anvil and suggests that the measured decreases in distance values may have been even greater with more aggressive cleaning of surfaces.

Morphometric analyses of the surfaces (Table 1) show that the slope, roughness and curvature of stone hammers and anvils are all modified by percussion activities. The surfaces alternate between smooth and rough conditions. As the surface degrades it becomes smoother due to the crushing of local peaks, while a rougher surface can also develop due to cracking. However, the trend is towards smoother surfaces overall as is demonstrated by the low slope and roughness values in stage 6 of $98 \mathrm{CP} 2$ compared with the measurements of this $\mathrm{CP}$ before modification.

\subsection{Implications for the analysis of archaeological percussive artefacts}

Ollé and Vergès (2014) stressed that use-wear must be observed with a range of magnifications throughout its development to evaluate the formation of these traces on stone tools. Our use of a high-resolution laser scanning confocal microscope and a low magnification stereo microscope follows this prescription, while also providing quantitative data on changes to surface morphology after the repeated use of pounding tools. The results based on the micro-3D scanning are consistent with those obtained with the stereo microscope in reflecting a low degree of modification in the surfaces of pounding tools that were used in bone breaking activities. Both techniques also demonstrate that use-wear formation is progressive and that raw materials of the hammerstone and anvil likely influence how fast and extensive use-wear is developed.

The results of our analyses suggest hominin breakage of limb bones using a 
hammerstone-on-anvil technique would be difficult to detect on pounding tools in the archaeological record. When damage is severe enough to be perceivable using a stereo microscope or even a naked eye, it would likely be mistaken with that created on hammerstones during stone flake production. This suggests many seemingly unmodified cobbles and blocks at archaeological sites could be minimally used pounding tools with only minor use-wear traces.

The experimental analysis of pounding tools can help us understand use-wear formation processes. The micro-3D analyses used here have revealed traces that were not observable with traditional methods, highlighting the potential of this technology in use-wear analysis. However, while the quantification of microscopic use-wear using 3D modeling generates reliable data that can be used to assess use-wear formation processes, a proper understanding of the development of use-wear on experimental tools is necessary to appreciate these changes. Lastly, modifications produced through natural processes must also be considered when applying this technique to archaeological material.

\section{Conclusions}

This paper develops a new method to quantify microwear traces on pounding tools using 3D techniques to measure high-resolution 3D data collected with a confocal microscope. Results show that microwear is slow to develop during bone breaking activities, yet small changes in surface topography are measureable using micro-3D data. In general, surfaces of tools become smoother with use although surface morphology can alternate between rougher and smoother states due to the development of microfractures. These changes are amplified in the active element (hammerstone) compared with the passive element (anvil). Use-wear on the active elements occurred over a larger area than on the passive elements, but the latter often exhibited deeper modifications. 
Understanding the development of percussive activities over the course of human evolution has the potential to provide important implications about the behavior of our ancestors and their subsistence strategies. At Olduvai Gorge (Tanzania) research has shown a great diversity of percussive artefacts identified through the archaeological sequence (from Bed I to Bed IV) (Leakey, 1971; Jones, 1994; Mora and de la Torre, 2005), and experimental programs have since focused on setting up protocols to infer the use of these artefacts. Up until now, these studies have focused on macroscopic use-wear traces (de la Torre et al., 2013), and applying these protocols to the analysis of archaeological pounding tools (Arroyo and de la Torre, 2016). Here we provide a new protocol for the use of microscopic traces in these analyses and hope that future experiments will apply this protocol to characterize additional pounding activities.

\section{Acknowledgments}

We thank T. Proffitt and J. di Cerbo for their help during the experiments, and an anonymous reviewer for their comments, which served to improve this manuscript. Research at Olduvai Gorge by the Olduvai Geochronology Archaeology Project (OGAP) is authorized by the Tanzania Commission for Science and Technology (COSTECH), the Tanzanian Department of Antiquities, and the Ngorongoro Conservation Area Authority. Funding from the Leverhulme Trust (IN-052) and the European Research Council-Starting Grants (ORACEAF, project number 283366) are acknowledged.

\section{Bibliography}

Abellán, A., Calvet, J., Vilaplana, J.M., Blanchard, J., 2010. Detection and spatial prediction of rockfalls by means of terrestrial laser scanner monitoring. Geomorphology, 119(34), $162-171$. 
Álvarez, M., Fuentes, N.O., Favret, E.A., Vanina Dolce, M., Forlano, A., 2012. Quantifying use-wear traces through RIMAPS and variogram analyses. Archaeological and Anthropological Sciences, 4(2), 91-101.

Anderson, P., Astruc, L., Vargiolu, R., Zahouani, H., 1998. Contribution of quantitative analysis of surface states to a multi-method approach for characterizing plantprocessing traces on flint tools with gloss, in: Facchini, D., Palma di Cesnola, A., Piperno, M., Peretto, C. (Eds.). Functional analysis of lithic artefacts: current state of the research. Proceedings of the XIII Congress (Forli, 8-14 September 1996). UISPP. Volume 6 Tome II, Forli: ABACO, pp. 1151-1160.

Anderson-Gerfaud, P., 1981. Contribution méthodologique à l'analyse des microtraces d'utilisation sur les outils préhistoriques. Thèse de 3ème siecle. Université de Bordeaux.

Arroyo, A., Torre, I. de la, 2016. Assessing the function of pounding tools in the Early Stone Age: A microscopic approach to the analysis of percussive artefacs from Beds I and II, Olduvai Gorge (Tanzania). Journal of Archaeological Science, 74, 23-34.

Barceló, J.A., Pijoan, H., Vicente, O., 2001. Image quantification as archaeological description, in: Stancic, Z., Veljanovski, T. (Eds.). CAA'2000 computing archaeology for understanding the past. BAR International Series 931, Oxford, pp. 69-77.

Benito-Calvo, A., Carvalho, S., Arroyo, A., Matsuzawa, T., Torre, I. de la, 2015. First GIS analysis of modern stone tools used by wild chimpanzees (Pan troglodytes verus) in Bossou, Guinea, West Africa. Plos One 10(3), e0121613. doi:10.1371/journal.pone.0121613

Beyries, S., Delamare, F., Quantin, J.C., 1988. Tracéologie et regosimétrie tridimensionelle., in: Beyries, S. (Ed.). Industries Lithiques: Tracéologie et Technologie, vol 2. BAR International Series, Oxford, pp. 115-132. 
Blumenschine, R.J., 1988. An experimental model of the timing of hominid and carnivore influence on archaeological bone assemblages. Journal of Archaeological Science, $15(5), 483-502$.

Caruana, M.V., Carvalho, S., Braun, D.R., Presnyakova, D., Haslam, M., Archer, W., Bobe, R., Harris, J.W.K., 2014. Quantifying traces of tool use: A novel morphometric analysis of damage patterns on percussive tools. Plos One, 9(11), e113856. doi:10.1371/journal.pone.0113856

Conrad, O., Bechtel, B., Bock, M., Dietrich, H., Fischer, E., Gerlitz, L., Wehberg, J., Wichmann, V., Böhner, J., 2015. System for Automated Geoscientific Analyses (SAGA) v. 2.1.4, Geoscientific Model Development 8, 1991-2007.

Evans, A. A., Donahue, R. E., 2008. Laser scanning confocal microscopy: a potential technique for the study of lithic microwear. Journal of Archaeological Science, 35(8), 2223-2230.

Frison, G. C., 1968. A functional analysis of certain chipped stone tools. American Antiquity $33(2), 149-155$.

Girardeau-Montaut, D., 2015. CloudCompare. 3D point cloud and mesh processing software Open Source Project, 2.6 ed.

González-Urquijo, E., Ibáñez-Estévez, J. J., 2003. The quantification of use-wear polish using image analysis. First results. Journal of Archaeological Science, 30(4), 481489.

Grace, R., Graham, I. D. G., Newcomer, M., 1985. The quantification of microwear polishes. World Archaeology, 17(1), 112-120.

Hurcombe, L. M., 1992. Use-wear analysis and obsidian: theory, experiment and results, JR. Collins Publications.

Ibáñez-Estévez, J.J., Anderson, P., González-Urquijo, E., Gibaja Bao, J.F., 2016. Cereal 
cultivation and domestication as shown by microtexture analysis of sickle gloss through confocal microscopy, Journal of Archaeological Science 73, 62-81.

Jones, P. R., 1994. Results of experimental work in relation to the stone industries of Olduvai Gorge, in: Leakey, M. D., Roe, D.A. (Eds.). Olduvai Gorge. Excavations in Beds III, IV and the Masked Beds, 1968-1971. Cambridge University Press, Cambridge, pp. 254-298.

Kato, K., 2002. Classification of wear mechanisms/models. Journal of Engineering Tribology, 216(6), 349-355.

Keeley, L. H., 1980. Experimental determination of stone tool uses: a microwear analysis. University of Chicago Press, Chicago.

Keller, C. M., 1966. The development of edge damage patterns on stone tools. Man, 1(4), $501-511$

Kimball, L., Allen, P., Kimball, J., Schlichting, B., Pham, K., 1998. The analysis of microwear polishes with the atomic force microscope, in: Facchini, F., Palma di Cesnola, A., Poperno, M., Peretto, C. (Eds.). Functional analysis of lithic artefacts: current state of the research. Proceedings of the XIII Congress (Forli, 8-14 september 1996). UISPP. Volume 6 Tome II, Forli: ABACO, pp. 1121-1132.

Konschak, H., 2012. NanoFocus operation manual. Oberhausen.

Knutsson, K., 1988. Patterns of tools use. Scanning electron microscopy of experimental quartz tools. Societas Archaeologica Upsaliensis, Uppsala.

Lague, D., Brodu, N., Leroux, J., 2013. Accurate 3D comparison of complex topography with terrestrial laser scanner: Application to the Rangitikei canyon (N-Z), ISPRS. Journal of Photogrammetry and Remote Sensing, 82, 10-26.

Leakey, M. D., 1971. Olduvai Gorge, Vol. 3. Excavations in Beds I and II, 1960-1963. Cambridge University Press, Cambridge. 
Mansur-Franchomme, M. E., 1983. Scanning Electron Microscopy of dry hide working tools: the role of abrasives and humidity in microwear polish formation. Journal of Archaeological Science, 10(3), 223-230.

Mcdonald, D. A., 2014. The application of focus variation microscopy for lithic use-wear quantification, Journal of Archaeological Science, 48, 26-33.

Monserrat, O., Crosetto, M., 2008. Deformation measurement using terrestrial laser scanning data and least squares 3D surface matching. Theme Issue: Terrestrial Laser Scanning, $63,142-154$.

Mora, R., Torre, I. de la, 2005. Percussion tools in Olduvai Beds I and II (Tanzania): Implications for early human activities. Journal of Anthropological Archaeology, 24(2), 179-192.

Odell, G. H., 1981. The mechanics of use-breakage of stone tools: some testable hypotheses. Journal of Field Archaeology, 8(2), 197-209.

Ollé, A., Vergès, J. M., 2014. The use of sequential experiments and SEM in documenting stone tool microwear. Journal of Archaeological Science, 48, 60-72.

Olsen, M. J., Johnstone, E., Driscoll, N., Ashford, S. A., Kuester, F., 2009. Terrestrial laser scanning of extended cliff sections in dynamic environments: Parameter analysis. Journal of Surveying Engineering, 135(4), 161-169.

Plisson, H., 1985. Étude fonctionnelle dòtillages lithiques préhistorique par l'analyse des micro-usures: recherche méthodologique et archéologique. Paris: Université de Paris I. Panthéon Sorbone.

Sánchez Yustos, P., Diez- Martín, F., Díaz, I. M., Duque, J., Fraile, C., Domínguez, M., 2015. Production and use of percussive stone tools in the Early Stone Age: Experimental approach to the lithic record of Olduvai Gorge, Tanzania. Journal of Archaeological Science Reports, 2, 367-383. 
Schürch, P., Densmore, A. L., Rosser, N. J., Lim, M., Mcardell, B. W., 2011. Detection of surface change in complex topography using terrestrial laser scanning: Application to the Illgraben debris-flow channel. Earth Surface Processes and Landforms, 36(14), 1847-1859.

Semenov, S.A., 1964. Prehistoric technology: an experimental study of the oldest tools and artefacts from traces of manufacture and wear. Redwood Press Limited, Great Britain.

Stemp, W. J., Morozov, M., Key, A. J. M., 2015. Quantifying lithic microwear with load variation on experimental basalt flakes using LSCM and area-scale fractal complexity (Asfc). Surface Topography: Metrology and Properties, 3(3), 1-20.

Stemp, W. J., Lerner, H., Kristant, E. H., 2013. Quantifying microwear on experimental Mistassini quartzite scrapers: Preliminary results of exploratory research using LSCM and scale-sensitive fractal analysis. Scanning, 35(1), 28-39.

Stemp, W. J., Stemp, M., 2001. UBM laser profilometry and lithic use-wear analysis: A variable length scale investigation of surface topography. Journal of Archaeological Science, 28(1), 81-88.

Stemp, W. J., Stemp, M., 2003. Documenting stages of polish development on experimental stone tools: surface characterization by fractal geometry using UBM laser profilometry. Journal of Archaeological Science, 30(3), 287-296.

Stemp, W.J., Watson, A.S., Evans, A.A., 2016. Surface analysis of stone and bone tools, Surface Topography: Metrology and Properties, 4, DOI: 013001.10.1088/2051$672 X / 4 / 1 / 013001$

Stevens, N., E., Harro, D.R., Hicklin, A., 2010. Practical quantitative lithic use-wear analysis using multiple classifiers, Journal of Archaeological Science, 37, 2671-2678.

Sussman, C., 1988. A microscopic analysis of use-wear and polish formation on experimental 
quartz tools. BAR International Series 395, Oxford.

Torre, I. de la, Benito-Calvo, A., Arroyo, A., Zupancich, A., Proffitt, T., 2013. Experimental protocols for the study of battered stone anvils from Olduvai Gorge (Tanzania). Journal of Archaeological Science, 40(1), 313-332.

Toselli, A., Pijoan-López, J., Barceló, J. A., 2002. La descripción de las trazas de uso en materias primas volcánicas: resultados preliminares de un análisis estadístico descriptivo, in: Clemente Conte, I., Risch, R., Gibaja Bao, J.F. (Eds.). Análisis funcional. Su aplicación al estudio de las sociedades prehistóricas. Bar International Series 1073, Oxford, pp. 65-78.

Tringham, R., Cooper, G., Odell, G. H., Voytek, B., Whitman, A., 1974. Experimentation in the formation of edge damage: a new approach to lithic analysis. Journal of Field Archaeology, 1(1/2), 171-196.

Vaughan, P. C., 1985. Use-wear analysis of flaked stone tools. University of Arizona Press, Tucson.

Vergès, J. M., Ollé, A., 2011. Technical microwear and residues in identifying bipolar knapping on an anvil: experimental data. Journal of Archaeological Science, 38(5), 1016-1025.

Yamada, S., 1993. The formation process of use-wear polishes, in: Anderson, P., Beyries, S., Otte, M., Plisson, H. (Eds.), Traces et fonction: les gestes retrouvés: actes du colloque international de Liège 8-9-10 décembre 1990. University of Liège, Liège, pp. 433446.

Zhang, Z., 1994. Iterative point matching for registration of free-form curves and surfaces. International Journal of Computer Vision, 13(2), 119-152.

\section{List of Figures}


Figure 1. General view of the experiments (A), close up of fractured bone (B) and example of the selection of the area to be utilized and identification of control points (C) (CPs recorded using the binocular microscope are represented by dots, while those recorded with the 3D scanner are represented by squares).

Figure 2. The wear formation sequence on the anvil. Four control points (A to D) across the six stages were recorded throughout the breakage of twenty limb bones over six experimental sessions. Photos of CP-A were taken at $45 \times$ (scales: $500 \mu \mathrm{m}$ ), CP-B at $25 \times$ (scales: $1 \mathrm{~mm}$ ), CP-C at $20 \times$ (scales: $1 \mathrm{~mm}$ ) and CP-D at $30 \times$ (scales: $1 \mathrm{~mm}$ ). Note the superficial crystal crushing appreciable in the last stage (ST6) at CP-A and CP-B, while the other two control points did not show any significant modification. Photos from the unmodified state to ST5 were taken with the GX microscope, while the last series of images (ST6) were taken with the Leica microscope.

Figure 3. The wear formation sequence recorded in two control points (A and B) on the quartzite hammer O86 throughout the breakage of ten limb bones over three experimental sessions. Note the progressive development of the crushing in both control points, particularly in the last series of images (5). Photos 1 to 4 for both series were taken at $30 \times$ (scales: $1 \mathrm{~mm}$ ), while the last set of photos (5) were captured at $80 \times$ (scales: $500 \mu \mathrm{m}$ ). Photos from unmodified to ST2 were taken with the GX microscope, while the last series of images (photos 4-5) were taken with the Leica microscope.

Figure 4. The wear formation sequence on the basalt hammer O83 recorded in three control points (A to C), throughout the breakage of ten limb bones over three experimental sessions.

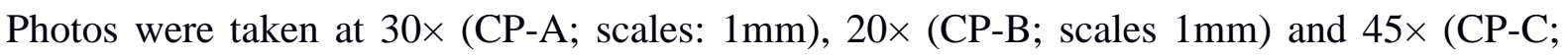
scales: $700 \mu \mathrm{m}$ ). Superficial crushing is visible in CP-B (ST-2 and ST-3), and small bone fragments can be seen embedded within the vesicles. Photos from unmodified to ST2 were 
taken with the GX microscope, while the last series of images (ST3) were taken with the Leica microscope.

Figure 5. Surface changes identified in pieces O83 and O98. A) Measured change considering the normal distance or separation between 3D point clouds UNM and ST3, estimated with algorithm M3C2. B) Areas displaying a statistically significant change, at 95\% confidence level. C) Histogram of significant changes measured with M3C2 algorithm. Surface changes detected in 98CP2 between stages UNM-ST3 (D and E: measured change considering the normal distance or separation between 3D point clouds UNM, ST3 and ST6, estimated with algorithm M3C2) and ST3-ST6 (F and G: Areas displaying a statistically significant change at $95 \%$ confidence level). H) Directional distributions of negative changes in pieces 98CP2 and 83CP1, generated during stages UNM-ST3 (98CP2 and 83CP1) and ST3-ST6 (98CP2).

\section{List of Tables}

Table 1. Mean values of the morphometric variables calculated for entire surface of the control points (CP) measured in pieces $\mathrm{O} 83$ and O98. Slope was calculated using the 2nd order polynomial method (Zevenbergen and Thorne, 1987). Profile curvature is calculated in the direction of the maximum slope, while plan curvature is measured perpendicular to the direction of the maximum slope. To estimate roughness, we applied the TRI and VRM indices (Benito-Calvo et al., 2015) calculated following Ridley et al. (1995) and Sappington et al. (2007).

Table 2. Area and percentage of the significant distance changes measured in the experimental pieces. A) Area and percentage of the surface significantly modified at a 95\% confidence level. B) Area and percentage of the negative and positive values detected in the 
areas of significant change.

\section{Supporting information}

Additional supporting information can be accessed in the online version of this paper at the publisher's web-site:

Table S1. Summary of the bone breaking experiments presented in this study.

Table S2. Registration errors during the alignment of the 3D point clouds.

Table S3. Parameters used to run the M3C2 algorithm. Numeric values are expressed in $\mu \mathrm{m}$.

Table S4. Metric used for measuring the spatial pattern of areas that demonstrated statistically significant changes (de la Torre et al., 2013). PA: Percentage of the area covered by use-wear. LUW (Largest use-wear): Percentage of the area covered by the largest usewear pattern. D (Density of battering marks): Number of battering marks divided by the total anvil area. ED (Edge Density): Total perimeter of the battering marks divided by the total anvil area. MNSH: Mean shape of the battering marks, comparing use-wear shapes with a standard circular shape (MNSH=1). Standard deviational ellipse: XstdD and YstdY, standard distances along short and long axes; Elongation, ellipse of the long axis divided by the short axis. Azimuth: Azimuth of the long ellipse axis.

Figure S1. Results of the slope (A) and curvature (B) analyses of each micro 3D control point before 3D alignment.

Figure S2. Results of the TRI (A and B) and VRM (C and D) roughness indices of each micro 3D control point before 3D alignment. Note the decrease-increase pattern seen on tools O83 and 086.

Figure S3. 3D Surface modifications on the basalt hammer and anvil. Images represent the unmodified surfaces (unm) and the last stage of use of CP1 on basalt hammer (O83) and CPs 2-3 on the anvil (O98). 